\title{
COVID-19: a short message to rheumatologists
}

\section{Piotr Leszczyński}

Department of Rheumatology and Rehabilitation, Poznań University of Medical Sciences, Poland Department of Rheumatology and Osteoporosis, Józef Struś Memorial Multidisciplinary Municipal Hospital

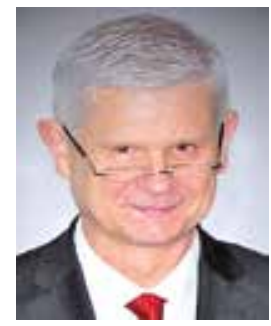
in Poznań, Poland

\begin{abstract}
About the Author
Piotr Leszczyński, MD, PhD - Professor of Karol Marcinkowski Poznań University of Medical Sciences, specialized in clinical rheumatology and internal medicine, head of the Department of Rheumatology and Osteoporosis of Józef Struś Memorial Multidisciplinary Municipal Hospital in Poznań, Poland and current president of the Poznań Branch of Polish Society of Rheumatology. Supervisor and reviewer of many bachelor's, master's and doctoral dissertations. Chief executive of medical specializations in rheumatology. Leading author or co-author of 110 published scientific papers. Founder, main scientific initiator and long-standing chairman of "Healthy Bones and Joints" conference, which has had 11 editions since 2010.
\end{abstract}

For several months, SARS-COV-2 virus infection, which sometimes causes critical COVID-19 disease primarily with lung involvement, has been the one and only medical and scientific topic. The widespread occurrence of this type of coronavirus has led to a global pandemic and a huge social confusion that has had and will have a significant impact on our lives.

From the point of view of a specialist in rheumatology and internal medicine, currently performing duties of a medical doctor of infectious diseases in the department of rheumatology in a temporarily uniform hospital in Poznań, Poland, various questions and reflections arise.

Does coronavirus, which turned out to be the greatest celebrity of the modern world, have any connection with the field of rheumatology? Do patients with rheumatic diseases have a higher risk of being infected with SARS-CoV-2 virus and developing COVID-19 disease? Does the standard treatment of synthetic and biological drugs that modifies autoimmune multiorgan inflammation have such a negative impact on the immune system that it could be a threat to rheumatological patients?

The developing infection from the rheumatologist's point of view seems to be understandable both at the stage of the mechanism of coronavirus entry through the ACE-2 receptor, which is distributed in epithelial cells of various systems and organs, as well as the mechanism of intracellular endocytosis with uncontrolled viral replication. Similarly, a subsequent immune dysregulation mechanism is characterized by a decrease in the concentration of type I and III antiviral interferons and excessive activation of primarily Th2 lymphocytes and signal transmission to B lymphocytes, which leads to a cytokine storm with symptoms of rapidly progressing pneumonia. At each of these stages of the disease, drugs that have been widely used by rheumatologists for many years could be implemented, and up until the global era of SARS-CoV-2 these drugs were uncommon in clinical practice of infectious disease physicians.

Chloroquine or hydroxychloroquine may have an in vitro antiviral effect by increasing lysosomal pH as well as by causing ACE-2 receptor glycosylation disorders, which leads to inhibition of SARS-CoV-2 fusion and replication. Another postulated mechanism is the inhibition of coronavirus accumulation in the structure of the endoplasmic reticulum called the ER-Golgi intermediate compartment (ERGIC), as well as inhibition of the expression of phosphatidylinositol binding the clathrin protein into the PICALM complex taking an active part in the process of SARS-CoV-2 endocytosis [1-3]. Certainly, the mechanism of blocking signaling of toll-like

Address for correspondence:

Piotr Leszczyński, Department of Rheumatology and Osteoporosis, Józef Struś Memorial Multidisciplinary Municipal Hospital, 3 Szwajcarska St., 61-285 Poznań, Poland, e-mail: piotr_leszczynski@wp.pl

Submitted: 2.06.2020; Accepted: 4.06.2020 
receptors and pro-inflammatory cytokine outbreak well known from rheumatology is also applicable here.

Unfortunately, despite previous reports, currently conducted in vivo clinical observational studies have not yet confirmed the effectiveness and safety of this therapy in both the treatment and prevention of SARS-CoV-2 infection. Additionally, analyses of large international registries suggest a lack of clinical efficacy of anti-malarial drugs in the treatment of COVID-19, and even an increased risk of death or life-threatening ventricular arrhythmias [4]. However, this published data is very controversial and requires extensive scientific discussion. The results of randomized trials that will give us more insights on clinical efficacy should be expected in the near future.

The weakening of the acute phase response in COVID-19 disease may also be associated with the inhibition caused by Janus kinase inhibitors (baricitinib, ruxolitinib, fedratinib) of clathrin-dependent viral endocytosis to pneumocytes regulated by the Numb-associated kinase (NAK family) [5].

In the treatment of cytokine storm in COVID-19, there is a possibility of using a TNF alpha inhibitor (adalimumab) or IL-6 receptor inhibitors (tocilizumab, sarilumab) [6-8], which are currently being studied in randomized clinical trials in SARS-CoV-2 infected patients with signs and symptoms of rapidly progressing pneumonia. IL-1 (anakinra) inhibitor [9], mycophenolate mofetil, tofacitinib, or IVIG infusions, as well as colchicine have also been used in described individual cases or larger groups of patients (non-commercial clinical studies) [10].

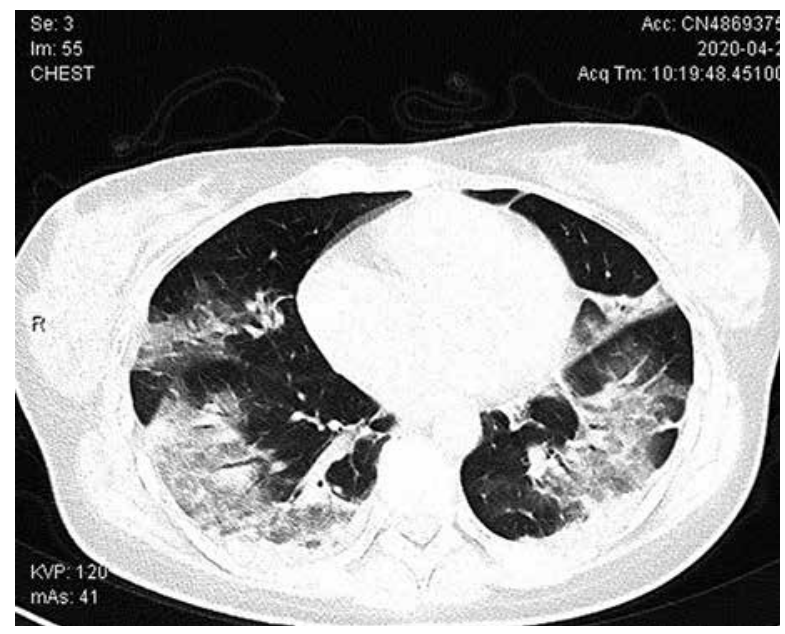

Fig. 1. A 30-year-old woman with severe COVID-19 pneumonia before treatment. HRCT performed on $21^{\text {st }}$ April, 2020.
Our own experience with the combined use of chloroquine and azithromycin or ceftriaxone $(n=34)$ and tocilizumab $(n=1)$ in the treatment of severe pneumonia in the course of COVID-19 disease is very good, although it should only be considered as a series of cases (Figs. 1 and 2, Table I). In our opinion, the treatment was effective and safe. Moreover, the analysis of the causes of death of our COIVID-19 patients $(n=3)$, especially in the context of the concurrent use of chloroquine and azithromycin, showed that only in one case at the time of death the patient received $250 \mathrm{mg}$ of chloroquine twice a day for 8 days, and no arrhythmias were found in this particular case.

In accordance with some clinical concerns of rheumatologists, patients with rheumatic diseases treated with disease-modifying drugs (DMARDs) should have a higher risk of SARS-CoV-2 infection. However, the first published studies from groups of patients from the region of Lombardy (Italy) and New York (USA) do not fully confirm these hypotheses. In the first case in the analyzed group of patients with rheumatic diseases $(n=320)$, only four cases of COVID-19 disease were confirmed. In the second case in the group of patients with ankylosing spondylitis, psoriatic arthritis and rheumatoid arthritis $(n=50)$ only one person died due to SARSCoV-2 active infection [11, 12]. In our sample of patients $(n=49)$ hospitalized in the rheumatology ward between March $9^{\text {th }} 2020$ and May $30^{\text {th }} 2020$ with suspected or diagnosed coronavirus infection, no case of a patient with rheumatic disease was found. Similarly, only 2 such cases from Poland were registered in the EULAR COVID-19 database as of May $26^{\text {th }} 2020$ [13].

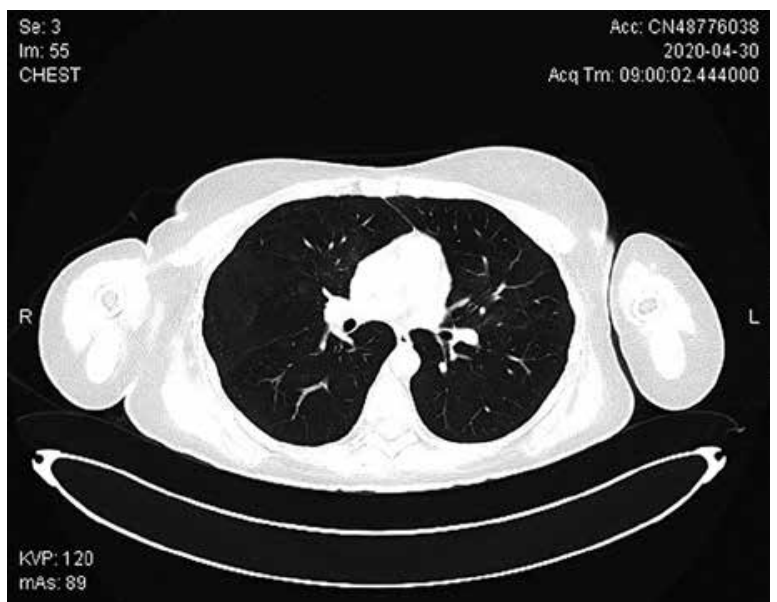

Fig. 2. A 30-year-old woman with severe COVID-19 pneumonia after treatment with chloroquine, azithromycin, lopinavir + ritonavir, tocilizumab, methylprednisolone for consecutive days. HRCT performed on $30^{\text {th }}$ April, 2020. 


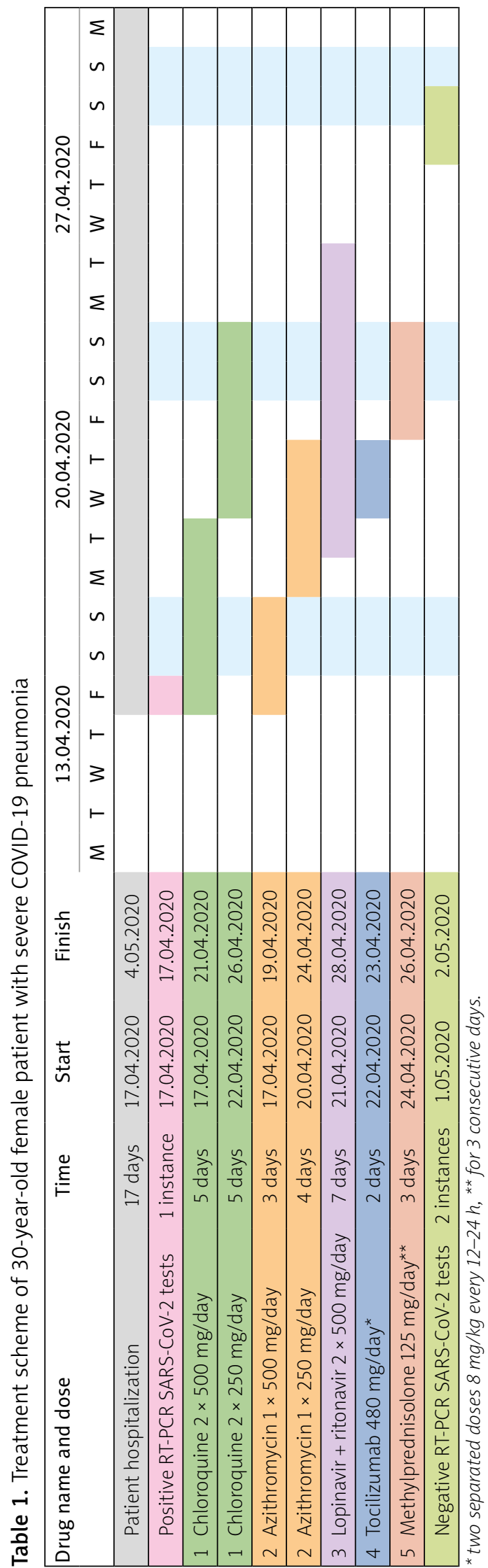

Perhaps dysregulation of the immune system due to generalized inflammation and the use of immunosuppressive and biological drugs that reduce the activity of the disease are in some sense potential factors protecting against the development of active infection. At present, it appears that patients with rheumatic diseases do not have a higher risk of developing SARS-CoV-2 infection, particularly severe, compared to the general population risk.

On April 27 2020 by consensus of 5 specialists in the field of rheumatology from the Poznan Branch of the Polish Society of Rheumatology the following guidelines for treating rheumatic patients in the time of COVID-19 were proposed:

1. Patient with pharmacologically treated rheumatic disease after close contact (staying at a distance of less than $2 \mathrm{~m}$, for more than 15 minutes, in the last 7 days) with a person with confirmed SARS-CoV-2 infection without clinical symptoms of COVID-19:

- maintain treatment with chloroquine/hydroxychloroquine, salazopyrin/sulfasalazine, corticosteroids, NSAIDs (if necessary),

- in anticipation of negative SARS-CoV-2 test results (RT-PCR only) or after two weeks without clinical signs of COVID-19, temporary discontinuation of methotrexate, leflunomide and biological disease-modifying drugs (very high doubt about standard drug withdrawal recommendations in this situation) and Janus kinase inhibitors (very high doubt about standard withdrawal recommendations in this situation due to the use of these drugs in clinical trials in patients with COVID-19) may be considered,

- use of tocilizumab should be continued; the drug is used to treat COVID-19 in selected clinical situations (off-label) and is currently being tested in numerous clinical trials.

2. Patient with pharmacologically treated rheumatic disease and documented SARS-CoV-2 infection with or without COVID-19 symptoms:

- maintain treatment with chloroquine/hydroxychloroquine, salazopyrin/sulfasalazine, corticosteroids, NSAIDs (if necessary),

- consult with the rheumatology department that treats patients with COVID-19,

- for patients with no clinical signs and symptoms of COVID-19, temporary discontinuation of methotrexate, leflunomide and biological disease-modifying drugs and Janus kinase inhibitors (tofacitinib, baricitinib) may be considered; at the same time, one should take into account the lack of an unambiguous standard of such proceedings, 
- refer patients with clinical symptoms of COVID-19 to the rheumatology ward that treats patients with COVID-19 and discontinue methotrexate, leflunomide and biological disease-modifying drugs as well as inhibitors of Janus kinases (tofacitinib, baricitinib); in the last case, one should have considerable doubt as to the standard of such proceedings with similar justification as above,

- in the absence of acute and very severe respiratory symptoms, it seems that the use of tocilizumab should be continued until hospitalization and further decisions.

Of course, the recommendations above are not based on strong scientific evidence, but solely on the analysis of currently available literature, reflection and own experience and should certainly be verified in the near future.

This time we are currently experiencing is very difficult, not only from a medical but also an organizational point of view. The celebrity virus has also reached the rheumatologists' environment, both in a purely scientific and also the most clinical context. However, we live with the hope that in Poland the time of COVID-19 is coming to an end and we will be able to return to our normal and everyday work in accordance with our medical specialization.

\section{Acknowledgments}

Many thanks for the hard work, from March $9^{\text {th }} 2020$ to the time of this text, to the whole Covid Rheumatology Team Poznań of my wonderful colleagues from the Rheumatology and Osteoporosis Department of the Multidisciplinary Municipal Hospital of Józef Struś in Poznań, Poland, who during the SARS-CoV-2 world pandemic had the opportunity to undergo solid training in the field of infectious diseases and passed this exam with flying colors: Katarzyna Pawlak-Buś, MD, PhD; Mateusz Kokot MD; Paula Kaczmarek MD; Cezary Iwaszkiewicz, MD, PhD; Wiktor Schmidt MD; Magdalena Owczarek MD; Kamila Gilewska MD and the Head of Nurses Anna Rewers MSc with the whole staff.

\section{References}

1. Savarino A, Boelaert JR, Cassone A, et. al. Effects of Chloroquine on Viral Infections: An Old Drug Against Today's Diseases? Lancet Infect Dis 2002, 3: 722-727, DOI: 10.1016/s14733099(03)00806-5.

2. Wolfram J, Nizzero S, Liu H, et al. A Chloroquine-Induced Macrophage-Preconditioning Strategy for Improved Nanodelivery. Sci Rep 2017, 7: 13738, DOI: 10.1038/s41598-017-14221-2

3. Hu YT, Frieman M, Wolfram J. Insights from nanomedicine into chloroquine efficacy against COVID-19. Nat Nanotechnol 2020: 1-3, DOI: 10.1038/s41565-020-0674-9 [Epub ahead of print].

4. Mehra MR, Desai SS, Ruschitzka F, Patel AN. Hydroxychloroquine or chloroquine with or without a macrolide for treatment of COVID-19: a multinational registry analysis. 2020, https://doi.org/10.1016/ S0140-6736(20)31180-6.

5. Richardson P, Griffin I, Tucker C, et al. Baricitinib as potential treatment for 2019-nCoV acute respiratory disease. Lancet 2020; 395: e30-e31, DOI: 10.1016/S0140-6736(20)30304-4.

6. Hoffmann M, Kleine-Weber H, Schroeder S, et al. SARS-CoV-2 cell entry depends on ACE2 and TMPRSS2 and is blocked by a clinically proven protease inhibitor. Cell 2020 (in press), DOI: 10.1016/j.cell.2020.02.052.

7. Xu X, Han M, Li T, et al. Effective treatment of severe COVID-19 patients with tocilizumab. ChinaXiv 2020, 202003.00026.

8. SIMIT - Societa`Italiana di Malattie Infettive e Tropicali Sezione Regione Lombardia. Vademecum per la cura delle persone con malattia da COVI-19. Edizione 2.0, 13 marzo 2020. http://www.simit.org/medias/1569-covid19- vademecum-13-03-202.pdf.

9. Sönmez HE, Demir S, Bilginer Y, Özen S. Anakinra treatment in macrophage activation syndrome: a single center experience and systemic review of literature. Clin Rheumatol 2018; 37: 3329-3335, DOI: 10.1007/s10067-018-4095-1.

10. Ferro F, Elefante E, Baldini C, et al. COVID-19: the new challenge for rheumatologists. Clin Exp Rheumatol 2020; 38: 175-180.

11. Monti S, Balduzzi S, Delvino P, et al. Clinical course of COVID-19 in a series of patients with chronic arthritis treated with immunosuppressive targeted therapies. Ann Rheum Dis 2020; pii: annrheumdis-2020-217424, DOI: 10.1136/annrheumdis-2020-217424 [Epub ahead of print].

12. Covid-19 in Immune-Mediated Inflammatory Diseases - Case Series from New York. Downloaded from nejm.org on May 10, 2020, DOI: 10.1056/NEJMc2009567.

13.https://www.eular.org/myUploadData/files/eular_covid_19_ registry_report.pdf.

The author declares no conflict of interest. 